\title{
Oxygen Pulse
}

National Cancer Institute

\section{Source}

National Cancer Institute. Oxygen Pulse. NCI Thesaurus. Code C123566.

The volume of oxygen consumed by the body per heartbeat while at rest. 